\title{
A Evolução do Capital Intelectual do IFBA no Período: 2009-2018
}

\author{
The Evolution of IFBA Intellectual Capital in the Period: 2009-2018
}

\author{
Zuneia Jesus Barros Reis ${ }^{1}$ \\ Elias Ramos de Souza ${ }^{1}$ \\ Nubia Moura Ribeiro ${ }^{1}$ \\ ${ }^{1}$ Instituto Federal da Bahia, Salvador, BA, Brasil
}

\begin{abstract}
Resumo
As instituições públicas brasileiras de ensino e pesquisa têm implementado políticas de incentivo à produção do conhecimento, com especial destaque para a composição do seu capital intelectual, com o propósito de contribuir com o desenvolvimento científico, tecnológico, econômico e social do país. Em particular, os Institutos Federais de Educação, Ciência e Tecnologia, criados em 2008, têm o intuito de realizar pesquisas aplicadas, estimulando o desenvolvimento de soluções técnicas e tecnológicas, estendendo seus benefícios à comunidade. O objetivo do presente estudo é analisar a evolução da qualificação do corpo docente e possível reflexo no panorama da produção científica e tecnológica do Instituto Federal de Educação, Ciência e Tecnologia da Bahia (IFBA), no período compreendido entre 2009 a 2018. Para tanto, foi realizado um levantamento na base Web Of Science e nos relatórios de gestão do IFBA. Metodologicamente essa pesquisa é de natureza quantitativa, do tipo descritiva, efetivada por meio de um estudo de caso. Os resultados da pesquisa permitiram concluir que houve uma evolução positiva na formação do capital humano, bem como no aumento da produtividade do conhecimento científico. Entretanto, registra-se uma acentuada queda da produtividade tecnológica institucional, evidenciada, sobretudo pela diminuição de registros ou depósitos de produtos tecnológicos no Instituto Nacional da Propriedade Industrial (INPI).

Palavras-chave: Institutos Federais de Educação. Ciência e Tecnologia. Capital Intelectual. Produção Científica e Tecnológica.
\end{abstract}

Área tecnológica: Propriedade Intelectual, Inovação e Desenvolvimento.

\begin{abstract}
Brazilian public educational and research institutions have implemented policies to encourage knowledge production, with special emphasis on their intellectual capital formation, aiming to contribute to the scientific, technological, economic and social development of the country. The Federal Institutes of Education, Science and Technology created in 2008 in particular, aim to carry out applied research, stimulating the development of technical and technological solutions, expanding their benefits to the community. The objective of this study is to analyze the qualification evolution of teaching staff and its possible repercussion on the panorama of the scientific and technological production of the Federal Institute of Education, Science and Technology of Bahia (IFBA), in the period from 2009 to 2018. To this end, a survey was conducted on the Web of Science database and IFBA management reports. Methodologically this is a quantitative research of descriptive type, carried out through a case study. The research results allowed to conclude that there was a positive evolution in the human capital formation, as well as in productivity increase of scientific knowledge. However, there was a sharp drop in institutional technological productivity, evidenced mainly by decrease in registrations or deposits of technological products at the National Institute of Industrial Property (INPI).

Key words: Federal Institutes of Education, Science and Technology. Intellectual capital. Scientific and Technological Production.
\end{abstract}

Technological Area: Intellectual Property. Innovation and Development. 


\section{Introdução}

A Lei n. 11.892, promulgada em 29 de dezembro de 2008, instituiu a Rede Federal de Educação Profissional, Científica e Tecnológica e criou os Institutos Federais de Educação, Ciência e Tecnologia (IFs), a partir da transformação e/ou integração das antigas Escolas Agrotécnicas, Escolas Técnicas Federais, Unidades Descentralizadas de Ensino, dos Centros Federais de Educação Tecnológica e das escolas vinculadas às universidades (BRASIL, 2008).

Os IFs são autarquias de regime especial, especializados na oferta de educação profissional e tecnológica, com atuação verticalizada, haja vista que atendem desde a formação básica de nível médio até a pós-graduação lato e stricto sensu. Do ponto de vista da produção do conhecimento, essas instituições têm como finalidade estimular o desenvolvimento de soluções técnicas e tecnológicas, estendendo seus benefícios à comunidade. Para o alcance dessa finalidade, a Lei atribuiu aos IFs a responsabilidade pela produção do conhecimento científico e tecnológico, com ênfase para o desenvolvimento da pesquisa aplicada em articulação com os diversos setores da economia, buscando promover o desenvolvimento socioeconômico local, regional e nacional (BRASIL, 2008).

As atividades de pesquisa institucionais no âmbito do Instituto Federal da Bahia - IFBA tiveram início nos anos 90, com a criação dos primeiros grupos de pesquisa no campus Salvador, a instituição do marco regulatório de estímulo à pesquisa científica e tecnológica, bem como a formação dos primeiros doutores da instituição. Contudo, foi a partir da transformação em Instituto Federal que a instituição avançou no tocante ao fortalecimento da infraestrutura de pesquisa em face da necessidade de atender aos desafios de elevação dos seus índices de produção do conhecimento (RAMOS DE SOUZA; RIBEIRO, 2009).

Um mapeamento da produção científica do IFBA em revistas de alto impacto foi apresentado por Ramos de Souza e Ribeiro (2009) em publicação comemorativa dos 100 anos da educação profissional no Brasil. Este estudo identificou uma tendência de crescimento da produção científica institucional e um claro crescimento anual do número de pesquisadores com titulação de doutorado, sinalizando o crescente interesse do IFBA em formar seu capital intelectual, elemento importante para o cumprimento da nova missão institucional.

Neste trabalho, a análise da produção científica e tecnológica do IFBA é retomada, considerando-se os primeiros 10 anos desde a criação do Instituto, ou seja, o período 2009 - 2018. Entretanto, o estudo aqui realizado vai além do levantamento da produção de artigos científicos realizado por Ramos de Souza e Ribeiro (2009) e aprecia também os produtos tecnológicos da Instituição na forma de patentes, programas de computador, desenhos industriais e marcas.

Na próxima seção (2) será apresentada uma breve revisão de literatura, seguida da metodologia na seção 3. A seção 4 é dedicada aos resultados e discussões e, finalmente, apresentam-se na seção 5 as considerações finais.

\section{Referencial Teórico}

Nahapiet e Goshal (1998) assinalam que os economistas tradicionalmente consideraram o capital físico e o capital humano como os recursos essenciais para as atividades produtivas e econômicas das firmas. Entretanto, o conhecimento passou a ser também reconhecido pelos 
economistas como um recurso valioso que constitui uma fração importante do capital empresarial. Neste sentido, emergiram disciplinas como a gestão do conhecimento e da inovação e desenvolveram-se novos conceitos, como o conceito de capital intelectual. Tendo sido definido de diferentes maneiras por vários autores, o capital intelectual pode ser considerado como o conhecimento e a capacidade de gerar ou adquirir novos conhecimentos de um coletivo social (NAHAPIET; GOSHAL, 1998). Com esta definição abrangente, deve-se considerar que o capital intelectual integra diferentes dimensões, dentre as quais se encontram os tipos de conhecimento. Em meados do século passado, Polanyi (1958) enfatizou que o conhecimento é pessoal e que as pessoas conhecem mais do que aquilo que conseguem expressar (POLANYI, 1962), o que levou ao estabelecimento dos conceitos de conhecimento tácito e conhecimento explícito, os quais têm sido fundamentais nas abordagens sobre a gestão do conhecimento e da inovação. Outras dimensões do capital intelectual têm sido consideradas e têm levado, inclusive, ao estabelecimento de métricas sobre o capital intelectual.

Bontis (2001) fez uma revisão da literatura sobre a mensuração do capital intelectual na qual identificou modelos que continuam figurando entre as principais referências utilizadas em gestão do conhecimento. O Navegador da Skandia, empresa de seguros e serviços financeiros da Escandinávia, adota um modelo que considera o capital intelectual como sendo a soma do capital humano e do capital estrutural. O IC-Index busca a consolidação de todos os indicadores individuais de capital intelectual em um único índice. O Technology Broker assume que o capital intelectual é um amálgama de quatro componentes: ativos de mercado, ativos humanos, ativos de propriedade intelectual e ativos de infraestrutura. O Monitor de Ativos Intangíveis se baseia em três famílias de ativos intangíveis: estrutura externa (marcas, clientes e fornecedores), estrutura interna (a organização e seus processos internos) e competência individual (educação, experiência). O modelo MVA e EVA, no qual o valor econômico adicionado (EVA) se destina a melhorar o valor adicionado de mercado (MVA), se baseia nas variáveis: orçamento, planejamento financeiro, metas, desempenho, comunicação aos acionistas e compensação de incentivos para contabilizar todas as maneiras pelas quais o valor corporativo pode aumentar ou diminuir. O método Citation-weighted Patents criado na Dow Química estima um "fator de tecnologia" para identificar o impacto dos esforços de $\mathrm{P} \& \mathrm{D}$ que levam à criação de propriedade intelectual e usa indicadores como investimentos em $\mathrm{P} \& \mathrm{D}$, número de patentes, custos de manutenção de patentes e custos dos projetos em relação à receita das vendas da empresa.

Perucchi e Mueller (2016) salientam que a produção do conhecimento nas sociedades modernas envolve instituições de ensino e pesquisa, empresas e governo e se desenvolve de maneira sinérgica, apesar de cada integrante possuir funções específicas, a saber: geração do conhecimento, aplicação do conhecimento em tecnologia e criação de políticas de estímulo, legislações e financiamentos. Nas últimas décadas, o Estado Brasileiro traduziu conceitos que emergiram na área de gestão do conhecimento e do capital intelectual em políticas públicas que se explicitaram no marco regulatório de ciência, tecnologia e inovação do país. A Lei n. 13.243, de 11 de janeiro de 2016, aprimorou políticas de estímulo ao desenvolvimento científico, à pesquisa, à capacitação científica e tecnológica e à inovação, instituídas a partir do início dos anos 2000, e introduziu, em seu artigo $2^{\circ}$, o conceito de capital intelectual como sendo o conhecimento acumulado pelo pessoal da organização, passível de aplicação em projetos de pesquisa, desenvolvimento e inovação (BRASIL, 2016). Ao mesmo tempo, estabeleceu diretrizes que formalizaram a importância do capital intelectual na dinâmica da produção científica, 
tecnológica e inovação, bem como a possibilidade de as instituições públicas de pesquisa compartilharem o seu capital intelectual com o setor produtivo.

As novas políticas de inovação apoiam-se fortemente nos fluxos de conhecimento, reconhecendo a absorção, internalização e geração do conhecimento como instrumentos essenciais no processo de inovação, capazes de ampliar o desempenho organizacional. De acordo com Lemos e Saraiva (2018), o capital intelectual é o conhecimento produzido pelas pessoas e principal ativo organizacional, pois é a partir dele que as organizações obtêm valor agregado. Assim, na visão dos autores, nenhuma atividade, seja material, tecnológica, financeira ou intelectual poderá ser produzida sem que seja utilizada alguma medida de capital intelectual.

Segundo Stewart (1998), o capital intelectual é um recurso intangível de uma organização, distinto pelo conhecimento que os colaboradores possuem e utilizam em suas atividades organizacionais. De outro modo, Edvinsson e Malone (1998) definem capital intelectual como a propriedade do conhecimento, experiência aplicada, tecnologia organizacional, relacionamento com clientes e habilidades profissionais que proporcionam vantagem competitiva de mercado.

É importante considerar que as distintas abordagens sobre capital intelectual enfatizaram a gestão do conhecimento em empresas. A sua extrapolação para outras formas de organização deve considerar as especificidades dos diferentes coletivos sociais. Neste trabalho, uma abordagem inicial sobre o capital intelectual de instituições científicas, tecnológicas e de inovação (ICTs) é realizada considerando-se a definição adotada na legislação brasileira (BRASIL, 2016), a qual pode ser interpretada utilizando-se o conceito de capital intelectual como sendo a soma dos capitais humano e estrutural (NAHAPIET; GOSHAL, 1998). Neste sentido, serão utilizados indicadores como o número de pesquisadores doutores, para descrição do capital humano, e as produções científica e tecnológica institucionais, na forma de conhecimentos explícitos (POLANYI, 1962), como uma proxy do capital estrutural.

\section{Metodologia}

Este trabalho caracteriza-se como uma pesquisa quantitativa, de caráter descritivo, efetivada por meio de um estudo de caso. Os dados relativos às publicações científicas foram coletados na base Web of Science, disponível no Portal de Periódicos da Coordenação de Aperfeiçoamento de Pessoal de Nível Superior (CAPES), por meio de pesquisa avançada em organização consolidada (OG) usando a expressão "Inst*Fed*da*Bahia".

A busca dos itens resultou em uma lista com 409 publicações científicas publicadas no interstício de 2009 a 2018. Os dados obtidos foram analisados considerando-se a evolução do número de publicações por ano, área do conhecimento, colaboração com outras instituições nacionais e estrangeiras, bem como mapa esquemático de países com publicações vinculadas ao IFBA, no período assinalado.

O mapeamento da produção tecnológica do IFBA foi realizado a partir de dados disponibilizados pelo Departamento de Inovação (Dinov), vinculado à Pró-Reitoria de Pesquisa, Pós-Graduação e Inovação (PRPGI) do IFBA e considerou a produção individual e coletiva e registros constantes do Instituto Nacional da Propriedade Industrial (INPI). Os relatórios de gestão institucionais foram analisados para fins de obtenção da evolução da formação do capital intelectual, especificamente quanto ao número de servidores com o título de doutor. 
Os dados obtidos foram utilizados para calcular as produtividades científica e tecnológica do IFBA. A produtividade científica é aqui definida como o número de publicações científicas por doutor por ano, enquanto que a produtividade tecnológica é o número de produtos tecnológicos por doutor por ano. Linhas de tendências da evolução anual das produtividades científica e tecnológica foram obtidas no Programa Excel (Office 2013). Em cada caso, foi escolhida a linha de tendência que apresentou maior coeficiente de determinação, $\mathrm{R}^{2}$, entre as opções das linhas de tendência exponencial, linear, logarítmica, polinomial e potência.

\section{Resultados e Discussão}

\subsection{Evolução do Capital Intelectual}

Para garantir o funcionamento de sua estrutura o IFBA possui em seu quadro o total de 2.552 servidores distribuídos nos cargos técnicos-administrativos (42\%) e docentes (58\%). Dentre os docentes, que são o foco da pesquisa, apenas 19,26\% possuem o título de doutorado. É importante destacar que ao longo do tempo o IFBA investiu em processos de qualificação dos seus docentes em programas de mestrado e doutorado patrocinados na forma de convênios, o que tem proporcionado a elevação do indicador de qualidade docente. Na última década, houve um aumento expressivo do quantitativo de docentes com titulação de doutorado, saindo de 81 doutores no ano de 2009 para 285 em 2018, conforme ilustra a Figura 1, o que corresponde a uma elevação em $250 \%$ do número de doutores.

Tendo em vista que o capital intelectual pode ser entendido como o conhecimento produzido pelas pessoas (LEMOS; SARAIVA, 2018), o aumento do número de doutores do IFBA é um claro indicador do potencial de aumento do capital intelectual da Instituição, posto que os pesquisadores doutores têm um papel central na produção de conhecimento científico $e$ tecnológico.

Figura 1 - Evolução anual do quantitativo de doutores

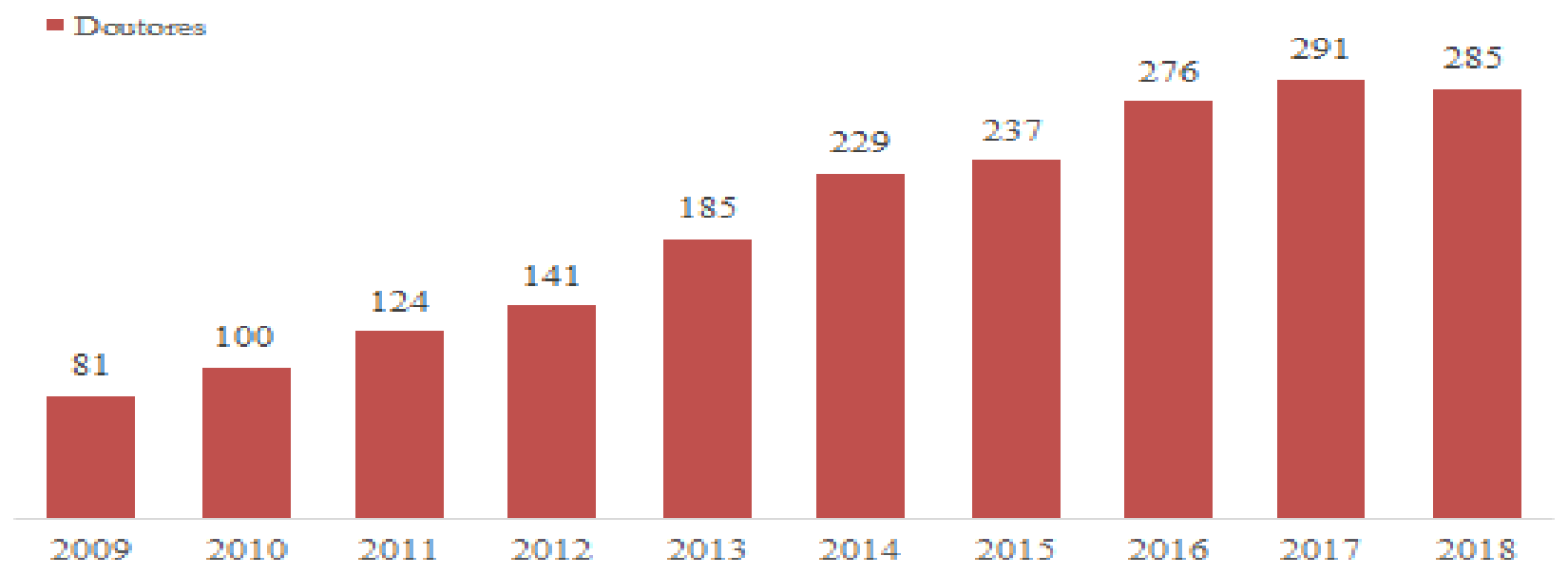

Fonte: IFBA (2018) 


\subsection{Produtividades Científica e Tecnológica}

O monitoramento do desenvolvimento de atividades de pesquisa científica e tecnológica no âmbito de uma instituição de ensino e pesquisa pode ser analisado por meio de inúmeros indicadores. Neste estudo, foram utilizados os indicadores de produtividade tecnológica associada ao quantitativo dos pedidos de depósito de patentes, marcas e registro de programas de computador referentes ao interstício 2009 - 2018, bem como a produtividade científica, evidenciada pelo número de publicações em revistas científicas de impacto internacional, no mesmo período, conforme a Figura 2.

Figura 2 - Evolução da produtividade científica do IFBA: 2009-2018

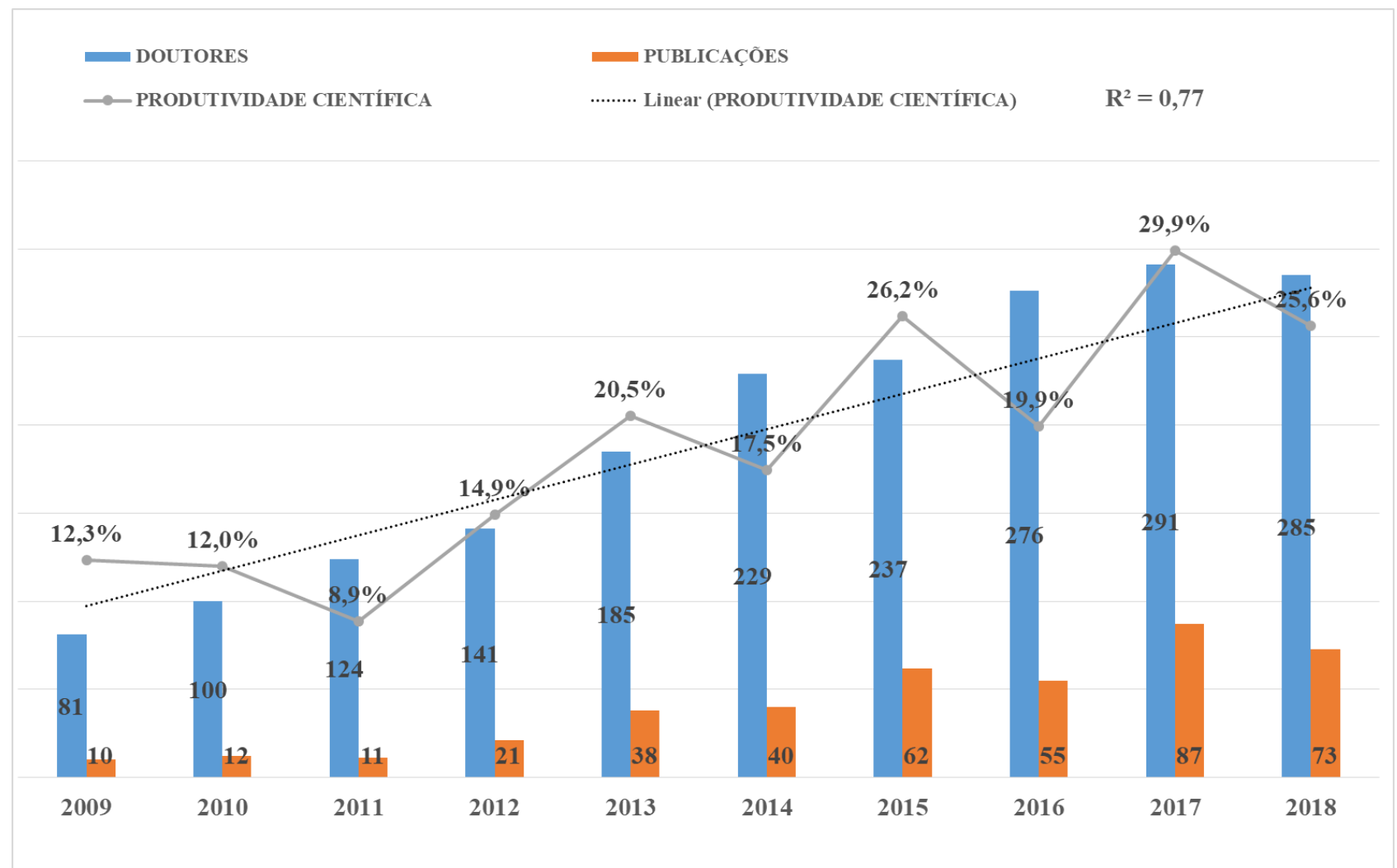

Fonte: Elaborada pelos autores deste artigo com base em: Web of Science; Portal de Periódicos da CAPES; Relatórios de Gestão do IFBA

Observou-se um tímido crescimento no número de publicações no período de 2009 a 2011. Porém, a partir de 2012, o quantitativo de publicações aumentou consideravelmente, com destaque para os anos de 2017 e 2018, que contou, respectivamente, com 87 e 73 publicações. Por sua vez, a produtividade científica dos doutores, medida como sendo o percentual de publicações por doutor por ano, também mostra crescimento anual, passando de 12,3\% em 2009 para 25,6\% em 2018. A produtividade científica praticamente dobrou ao longo do período, indicando tendência de crescimento aproximadamente linear (coeficiente de determinação $\mathrm{R}^{2}=0,77$ ).

No tocante às publicações em cooperação com instituições nacionais, destacam-se as estabelecidas com a Universidade Federal da Bahia, com 179 publicações, seguidas da Universidade Federal de Sergipe (27), Universidade Estadual de Santa Cruz (26), Empresa Brasileira de Pesquisa Agropecuária - EMBRAPA (22), Universidade Federal da Paraíba (20), Universidade 
de São Paulo (19), Universidade Federal do Rio Grande do Norte (18), Universidade Estadual do Sudoeste da Bahia (17) e Universidade Estadual da Bahia (16), segundo a Figura 3.

Figura 3 - Quantitativo de publicações do IFBA com organizações parceiras

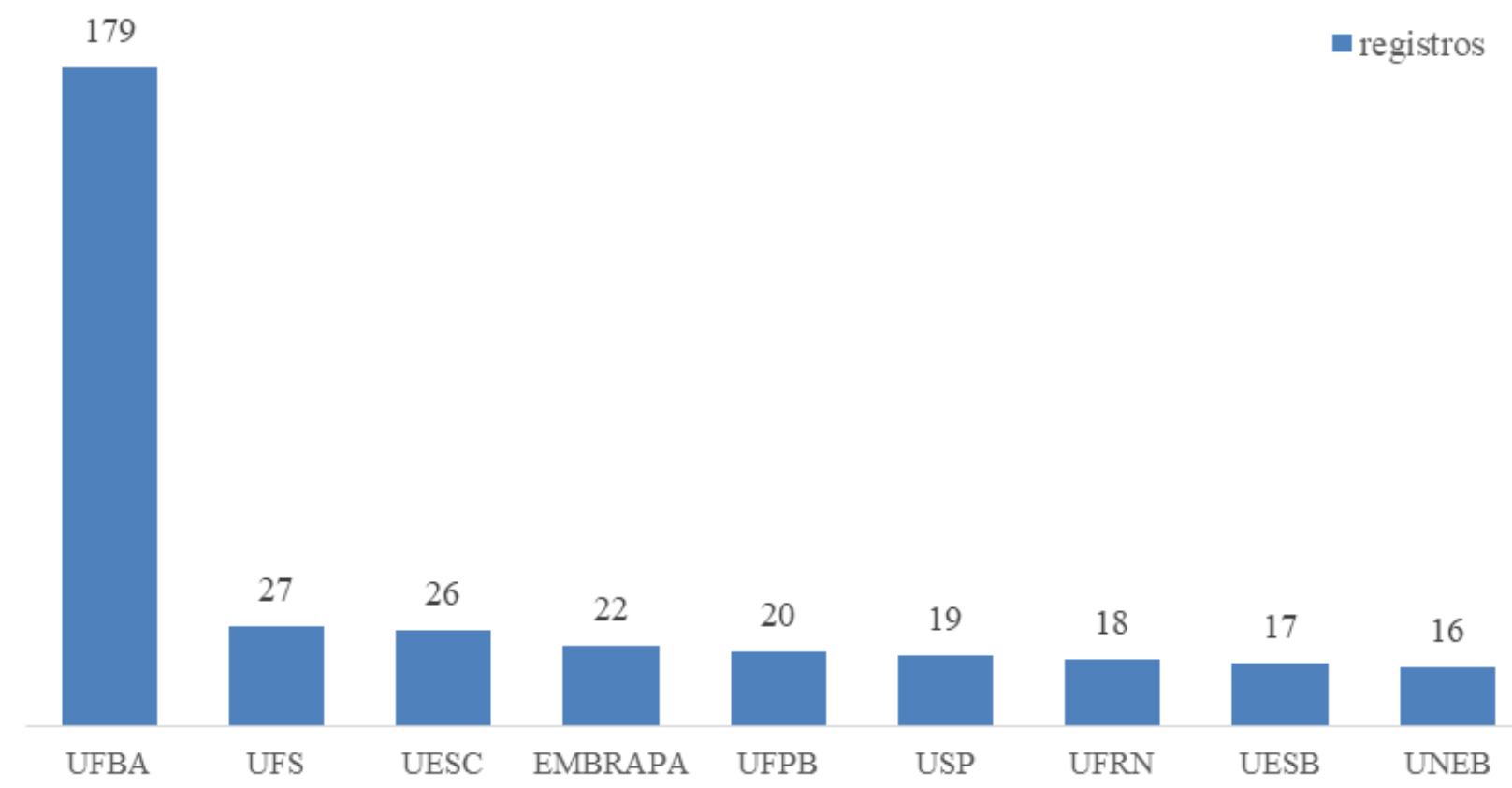

Fonte: Elaborada pelos autores deste capítulo com base em Portal CAPES (2019)

Outro achado importante é o mapa esquemático explicitado pela Figura 4, que apresenta os 10 países cuja afiliação dos autores demonstra maior número de publicações em colaboração com o Instituto Federal da Bahia, com destaque para os Estados Unidos, com 29 publicações; a Espanha, com 9 publicações; a Argentina, com 7 publicações; o Chile, com 6 publicações; seguidos por Irã e Ucrânia (5), Dinamarca, Inglaterra e Alemanha (3).

Figura 4 - Mapa esquemático dos países com publicações vinculadas ao IFBA

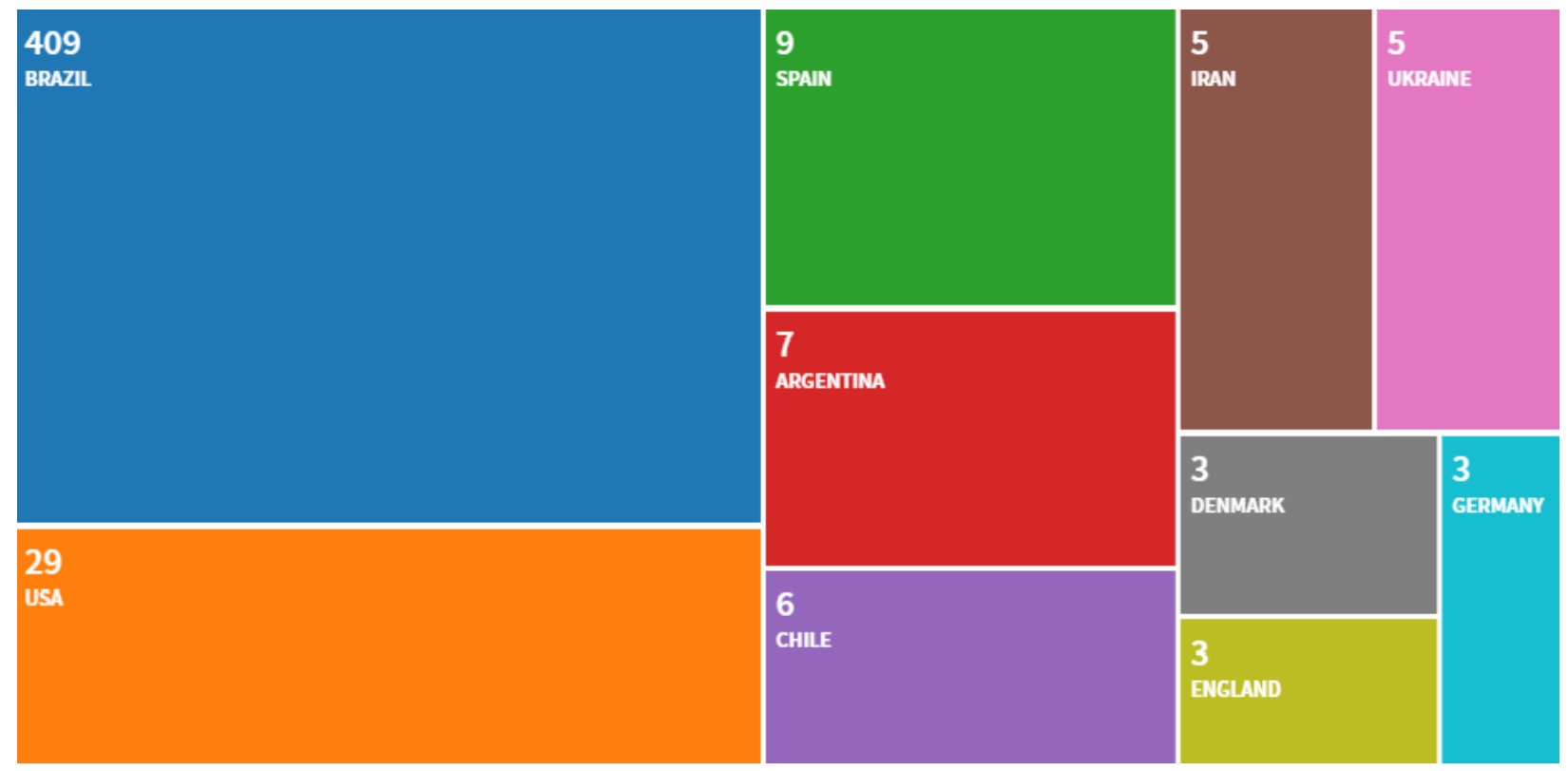

Fonte: Portal CAPES (2019) 
Ao filtrar as publicações por área de conhecimento, evidenciou-se uma concentração de produções nas áreas de engenharia, química, ciência da computação, física, agricultura, ciências ambientais e ecológicas, ciências materiais, tecnologias da ciência outros tópicos, biologia molecular bioquímica e ótica, conforme a Figura 5.

Figura 5 - Quantitativo de publicações X área do conhecimento

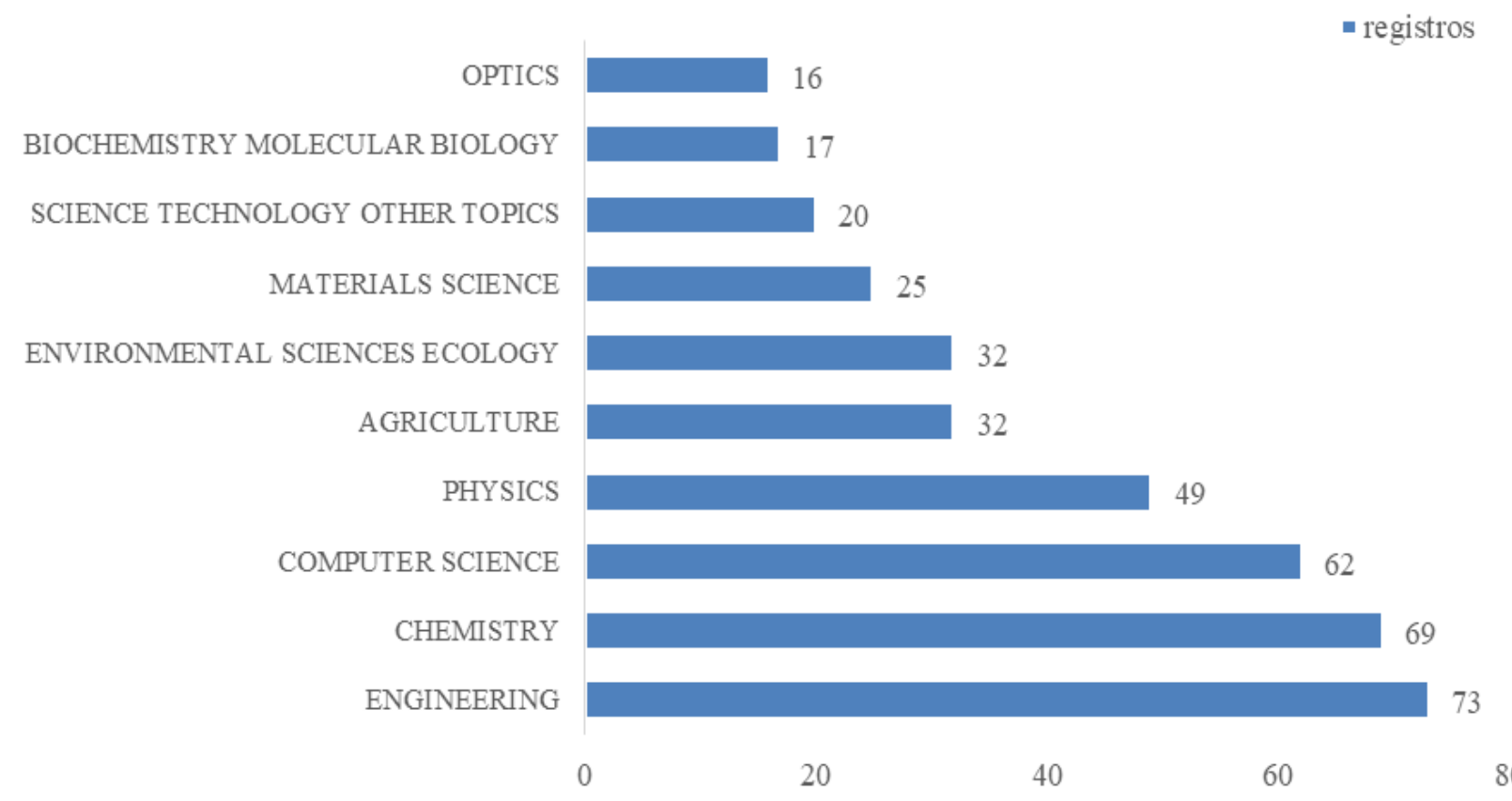

Fonte: Portal CAPES (2019)

No tocante à propriedade industrial proveniente de projetos de pesquisa, no período de 2009 a 2018, o IFBA depositou o total de 35 pedidos de patente de invenção (PI), 20 registros de software (SO), 8 patentes modelo de utilidade (MU) e 1 registro de desenho industrial (DI), consoante a Figura 6.

Figura 6 - Mapa de propriedade industrial do IFBA: 2009 a 2018

$$
\text { घatente de Invenção } \quad \text { Modelo de utilidade } \quad \text { Software } \quad \text { Desenho Industrial }
$$

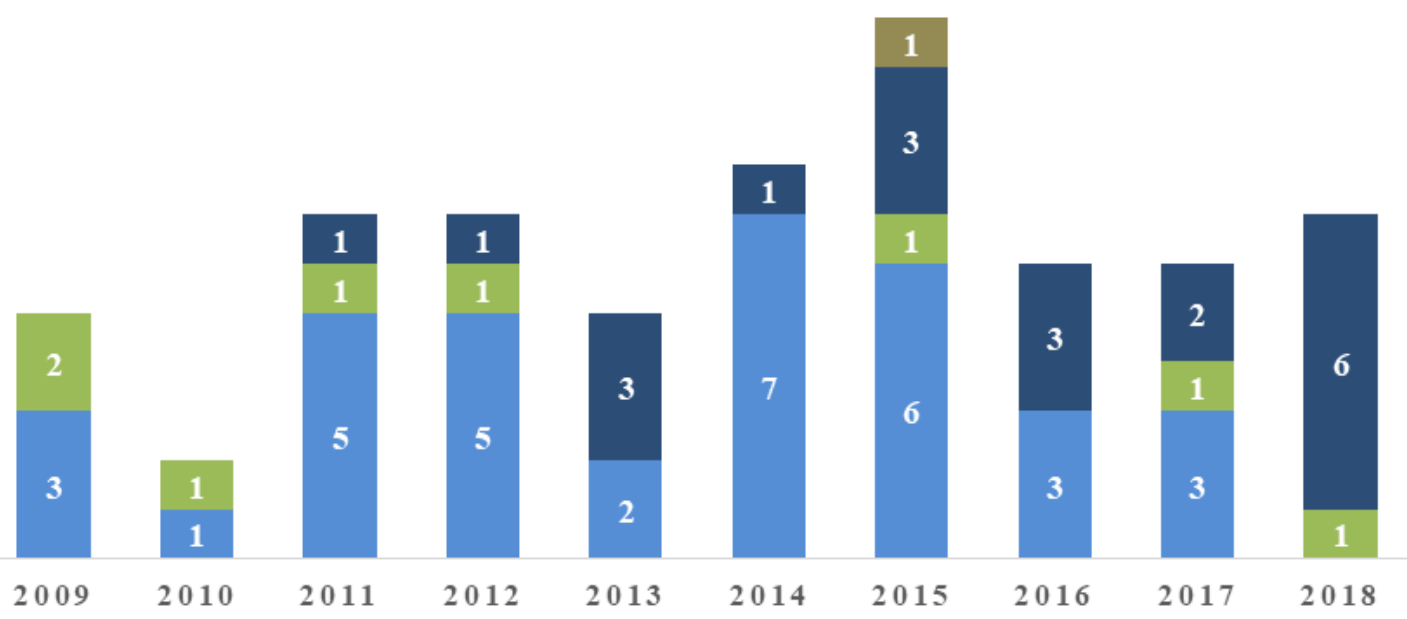

Fonte: Departamento de Inovação/IFBA (2019) 
Da análise do mapa de propriedade intelectual produzida pelos grupos de pesquisa do IFBA, depreende-se que há uma predominância de pedidos de patentes de invenção e registros de software, seguidos de patente de modelo de utilidade e desenho industrial, com destaque para os anos de 2014 e 2015 que contaram, respectivamente, com 8 e 11 pedidos.

A partir dos dados referentes aos produtos tecnológicos e do número de doutores obteve-se a evolução da produtividade tecnológica do IFBA no período 2009 - 2018, conforme a Figura 7. Os dados mostram uma queda acentuada da produtividade tecnológica, que era igual a $6,2 \%$ em 2009 e decresceu para 2,5\% em 2018. Neste caso, a linha de tendência linear apresentou baixo coeficiente de determinação $\left(R^{2}=0,34\right)$, o que é compatível com a sazonalidade apresentada pelo gráfico.

Figura 7 - Produtividade tecnológica do IFBA: 2009 a 2018

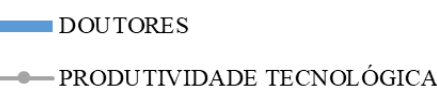

PRODUTOS TECNOLÓGICOS

$\longrightarrow$ PRODUTIVIDADE TECNOLÓGICA

…... Linear (PRODUTIVIDADE TECNOLÓGICA) $\quad \mathrm{R}^{2}=0,34$

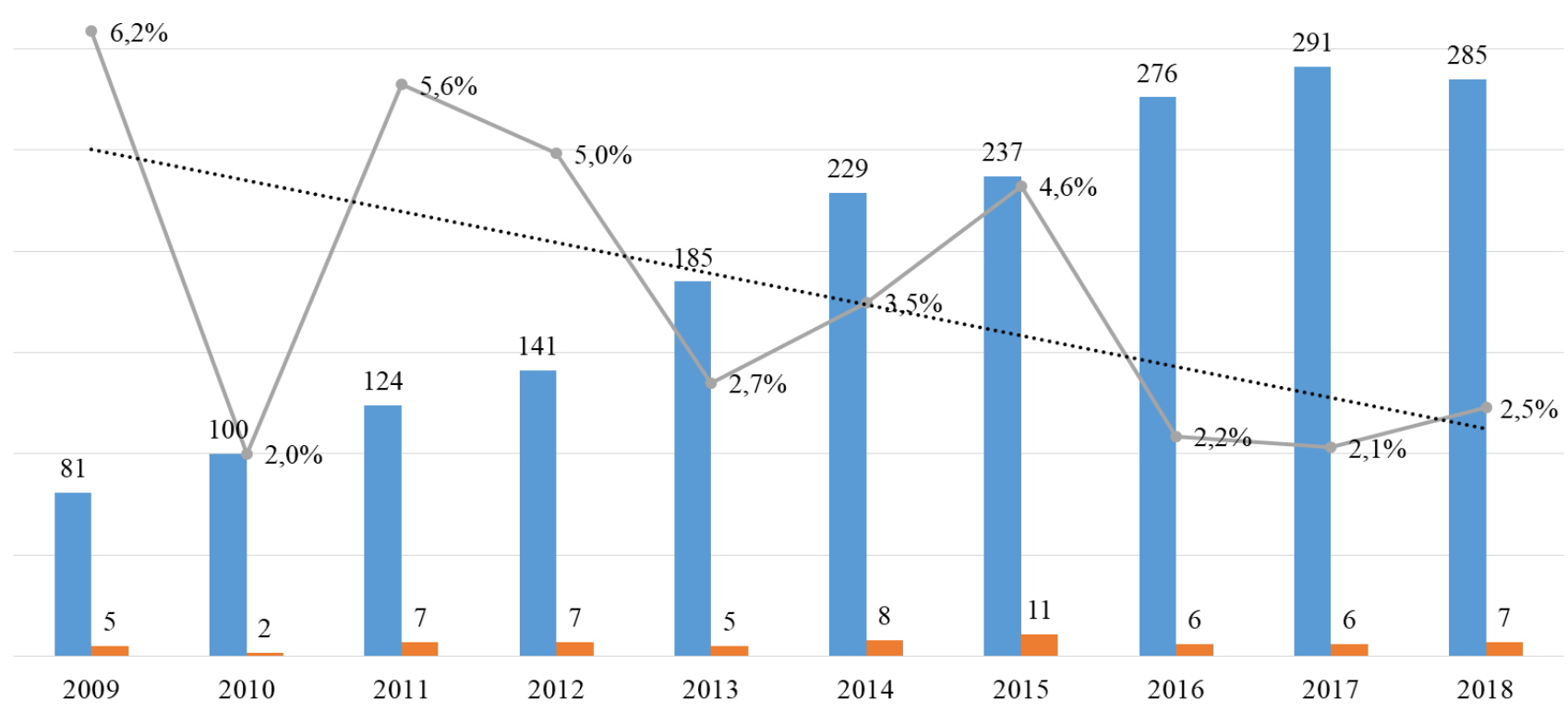

Fonte: Elaborada pelos autores deste artigo com base em: Departamento de Inovação/IFBA (2019); e Relatórios de Gestão do IFBA

Os resultados mostram, portanto, que enquanto a produtividade científica dobrou no período analisado a produtividade tecnológica decresceu, em 2018, para menos da metade do seu valor em 2009. Além desta discrepância entre as evoluções das produtividades científicas e tecnológicas, é importante notar que a produtividade científica é uma ordem de grandeza maior do que a produtividade tecnológica, estando a primeira na faixa de dois dígitos (25,6\% em 2018) enquanto que a segunda está na faixa de um dígito (2,5\% em 2018), de modo que a produtividade científica é cerca de dez vezes maior do que a produtividade tecnológica.

A Figura 8 mostra a evolução da razão entre os produtos tecnológicos e as publicações científicas, indicando uma queda ao longo do período, passando de 50\% em 2009 para 9,6\% em 2018. A linha de tendência exponencial foi a que apresentou maior coeficiente de determi- 
nação $\left(\mathrm{R}^{2}=0,64\right)$, indicando uma queda exponencial da relação entre produtos tecnológicos e produtos científicos no período.

Figura 8 - Evolução da razão entre os produtos tecnológicos e a produção científica do IFBA: 2009 a 2018

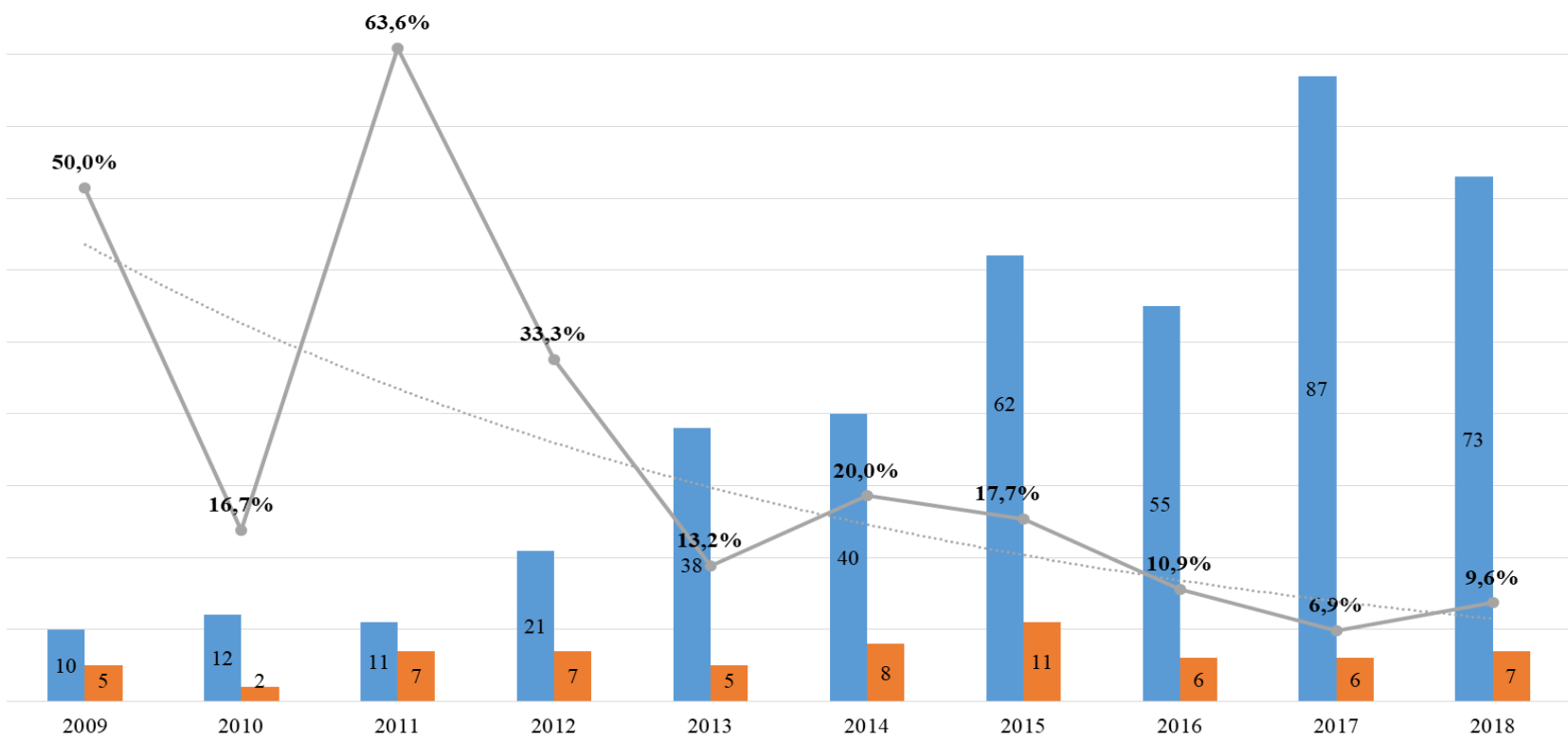

Fonte: Elaborada pelos autores deste artigo com base em: Departamento de Inovação/IFBA (2019); Web of Scicence; e Portal de Periódicos da CAPES (2019)

\section{Considerações Finais}

Os resultados descritos neste artigo demonstraram que há uma tendência de crescimento da produtividade científica do Instituto Federal de Ciência e Tecnologia da Bahia (IFBA), enquanto a produção tecnológica - medida pela quantidade de patentes, programas de computadores, desenhos industriais e marcas por ano - reduziu acentuadamente. Além disso, a razão entre as produções tecnológica e científica saiu de 50\% em 2009 para 10,1\% em 2018.

Evidenciou-se também a ampliação do quantitativo de doutores na ordem de $250 \%$ em relação ao ano de 2009. Esse dado revela a preocupação da instituição em formar seu capital intelectual a fim de cumprir suas novas atribuições no cerne do desenvolvimento científico e tecnológico do ambiente socioeconômico em que está inserida. As evoluções das produções científica e tecnológica comentadas acima podem estar associadas ao ingresso ou formação de novos doutores, cujos perfis poderão, em perspectiva, se ajustar à vocação da Instituição, mas é preciso que políticas institucionais sejam definidas para superar as distorções aqui identificadas.

O levantamento sobre pedido de registros de patentes demonstrou que o IFBA depositou o total de 71 pedidos de patentes de residentes junto ao INPI. Desses foram obtidas 02 (duas) cartas patentes de modelos de utilidade e 01 (um) de registro de desenho industrial. Percebe- 
-se ainda que a área das engenharias concentrou maior produção científica, com o total de 73 publicações, contrastando com os números apresentados no estudo realizado por Ramos de Souza e Ribeiro (2009), no qual a maior produção científica estava associada à área de química. Naquele estudo (RAMOS DE SOUZA; RIBEIRO, 2009) a área das engenharias ocupava a quarta posição com uma produção diminuta.

Verificou-se também a ampliação do número de publicações em cooperação com organizações nacionais e estrangeiras, com ênfase para a Universidade Federal da Bahia, que continua sendo a principal parceira no âmbito das universidades localizadas no Estado da Bahia. No cenário internacional destacou-se o número de publicações em colaboração com autores dos Estados Unidos, Espanha e Argentina.

Esses resultados são importantes, pois sinalizam que o instituto precisa desenvolver ações e políticas para estimular a interação entre as áreas de ensino, pesquisa e extensão, além de fortalecer estruturas internas com o intuito de facilitar a transferência do conhecimento científico para o ambiente empresarial.

\section{Referências}

BONTIS, Nick. Assessing knowledge assets: a reiew of the models used to measure intelectual capital. International Journal of Management Reviews, [S.l.], v. 3, n. 1, p. 41 - 60, 2001.

BRASIL. Lei n. 11.892, de 29 de dezembro de 2008. Institui a Rede Federal de Educação Profissional, Científica e Tecnológica. Cria os Institutos Federais de Educação, Ciência e Tecnologia e dá outras providências. Diário Oficial da União, Brasília, DF, 30 dez. 2008. Seção 1, p. 01. Disponível em: http://www.planalto.gov.br/ccivil_03/Ato2007_2010/2008/ Lei/L11892.htm. Acesso em: 10 fev. 2019.

BRASIL. Lei n. 13.243, de 11 de janeiro de 2016. Dispõe sobre estímulos ao desenvolvimento científico, à pesquisa, à capacitação científica e tecnológica e à inovação. Diário Oficial da União, Brasília, DF, 12 jan. 2016. Disponível em: http:/www.planalto.gov.br/ccivil_03/_ato2015-2018/2016/ lei/113243.htm. Acesso em: 10 fev. 2019.

EDVINSSON, Leif; MALONE, Michel S. Capital Intelectual. Tradução de Roberto Galman. São Paulo: Makron Books, 1998. Disponível em: http://capitalintelectual.egc.ufsc.br/wp-content/ uploads/2016/05/1997-Edvinsson-e-Malone.pdf. Acesso em: 20 fev. 2019.

INSTITUTO FEDERAL DA BAHIA. Relatório de gestão institucional 2013. Salvador, BA: IFBA, 2013. Disponível em: http://portal.ifba.edu.br/proap/transparencia-arquivos/relatorios-de-gestao-doifba/2013-relatorio-de-gestao-institucional.pdf/view. Acesso em: 05 jan. 2019.

INSTITUTO FEDERAL DA BAHIA. Relatório de gestão institucional 2015. Salvador, BA: IFBA, 2015. Disponivel em: http://portal.ifba.edu.br/proap/transparencia-arquivos/relatorios-de-gestao-doifba/2015-relatorio-de-gestao-institucional.pdf/view. Acesso em: 06 fev. 2019.

INSTITUTO FEDERAL DA BAHIA. Relatório de gestão institucional 2016. Salvador, BA: IFBA, 2016. Disponível em: http://portal.ifba.edu.br/proap/transparencia-arquivos/relatorios-de-gestao-doifba/2016-relatorio-de-gestao-institucional.pdf/view. Acesso em: 06 fev. 2019. 
noticias/2018/06/ifpb-e-o-primeiro-entre-os-institutos-federais-no-ranking-de-patentes-deinvencao/2018-ranking_res_pi_2017.pdf. Acesso em: 20 jan. 2019.

LEMOS, Alisson Ferreira de; SARAIVA, Myrella Marques. Práticas de gestão do conhecimento atreladas à aprendizagem organizacional: um estudo de caso. Revista Gestão em Foco, Amparo/SP, ed. 10, 2018. Disponível em: http://portal.unisepe.com.br/unifia/wp-content/uploads/sites/10001/2018/06/018_ PRÁTICAS_DE_GESTÃO_DO_CONHECIMENTO.pdf. Acesso em: 10 jan. 2019

NAHAPIET, J., GOSHAL, S. Social captal, intellectual capital, and the organization advantage. Academy of Management Review, [S.I.], v. 23, n. 2, p. 242-266, 1998.

PERUCCHI, Valmira; MUELLER, Suzana Pinheiro Machado. Produção de conhecimento científico e tecnológico nos Institutos Federais de Educação, Ciência e Tecnologia: uma investigação sobre a sua natureza e aplicação. Perspectivas em Ciência da Informação, [S.l.], v. 21, n. 1, p. 134151, jan./mar. 2016. Disponível em: http://portaldeperiodicos.eci.ufmg.br/index.php/pci/article/ view/2503/1716. Acesso em 10 jan. 2019.

RAMOS DE SOUZA, E.; RIBEIRO, N. M. A Produção Científica do Instituto Federal da Bahia em Revistas de Alto Impacto. In: FARTES, Vera; MOREIRA, Virlene Cardoso (Org.). Cem Anos de Educação Profissional no Brasil - História e Memória do Instituto Federal da Bahia (1909 -2009). 1. ed. Salvador: EDUFBA, 2009. p. 125-139.

POLANYI, Michael. Personal Knowledge: towards a post-critical philosophy. London: Routledge \& Kegan Paul Ltd., 1958.

POLANY, Michael. Tacit knowing: its bearing on some problems of philosophy. Reviews of Modern Physics, [S.I.], v. 34, n. 4, p. 601-616, 1962.

STEWART, Thomas A. Capital intelectual: a nova vantagem competitiva das empresas. 2. ed. Rio de Janeiro: Campus, 1998.

\section{Sobre os Autores}

\section{Zuneia Jesus Barros Reis}

E-mail: zuneiabr@hotmail.com

Mestra em Propriedade Intelectual e Transferência de Tecnologia para a Inovação

Endereço profissional: Instituto Federal de Educação, Ciência e Tecnologia da Bahia, Reitoria, Av. Araújo Pinho,

39, Canela, Salvador, BA, Brasil. CEP: 40110-150.

\section{Elias Ramos de Souza}

E-mail: eramosdesouza@gmail.com

Doutor em Biofísica

Endereço profissional: Instituto Federal de Educação, Ciência e Tecnologia da Bahia, Campus Salvador, Rua Emídio dos Santos, s/n, Barbalho, Salvador, BA, Brasil. CEP: 40301-015.

\section{Nubia Moura Ribeiro}

E-mail: nubiamouraribeiro@gmail.com

Doutora em Química

Endereço profissional: Instituto Federal de Educação, Ciência e Tecnologia da Bahia, Campus Jequié, Rua Jean Torres de Oliveira, s/n., Bairro John Kennedy, Loteamento Cidade Nova, Jequié, BA, Brasil. CEP: 45201-767. 